\title{
ESTRUCTURA OCUPACIONAL ARGENTINA. ¿QUÉ OCULTAN LOS PROMEDIOS? ANÁLISIS SEGÚN REGIONES Y ESCALA URBANA. AÑO 2010
}

\author{
Fernando Manzano ${ }^{l}$ \\ Guillermo Velázquez ${ }^{2}$
}

\begin{abstract}
RESUMEN
Existen ciertas características correspondientes a la inserción ocupacional que son consideradas como comportamientos regulares, pero que solo representan el resultado de la información del mercado laboral a nivel agregada, es decir, surgen como un promedio de situaciones muy heterogéneas. En este trabajo tomaremos tres afirmaciones que suelen esgrimirse frecuentemente como válidas como ser: mayor participación en el mercado laboral masculina que femenina; existencia de una relación positiva entre nivel educativo e intensidad en la participación económica de las personas; y baja inserción laboral de los grupos en edades más jóvenes y más avejentados. Si consideramos unidades menores de análisis, comienzan a cobrar importancia las diferentes características regionales que conforman el territorio argentino, así como también al interior de las mismas el grado de concentración urbana de cada área espacial bajo análisis. El objetivo de este trabajo es demostrar que mediante el uso de información más desagregada, pueden detectarse situaciones opuestas a las mencionadas anteriormente, que son de validez para una descripción al nivel más agregado.

Con respecto a la información estadística que utilizaremos en este artículo, corresponde al bloque ocupacional de los últimos tres Censos Nacionales de población en la Argentina, llevados adelante en los años 1991, 2001 y 2010.
\end{abstract}

Palabras Clave: Escalas urbanas; Participación; Laboral; Sexo

\section{OCCUPATIONAL STRUCTURE ARGENTINA WHAT HIDDEN AVERAGES? ANALYSIS ACCORDING TO REGIONS AND URBAN SCALE. YEAR 2010}

\section{ABSTRACT}

There are certain characteristics relevant to the occupational integration that are considered as regular behavior, but that only represent the result of the labor market information to aggregate level, i.e. arise as an average of situations very heterogeneous. In this work we will take three statements that often be wielded frequently as valid as being: greater participation in the labor market than a feminine male; existence of a positive relationship between educational level and intensity in the economic participation of the people; and low labor insertion of the groups in younger ages and younger age groups. If we consider smaller units of analysis, are beginning to gain importance the different regional characteristics that make up the Argentine territory, as well as within the same degree of urban concentration of each spatial area under analysis. The aim of this paper is to demonstrate that through the use of more disaggregated information, can be detected to the contrasting situations mentioned above, which are valid for a description to the most aggregate level.

With regard to the statistical information that will be used in this article, the occupational block for the last three national censuses of population in Argentina, brought forward in the years 1991, 2001 and 2010.

Keyword: Scales Urban; Participation; Labor; Sex

\footnotetext{
${ }^{1}$ Universidad Nacional de Córdoba. Rep. Argentina. Email: fernandoarielmanzano@ hotmail.com ${ }^{1}$ Instituto de Geografía, Historia y Ciencias Sociales (CONICET/UNCPBA). ${ }^{2}$ Universidad Nacional de Córdoba. Rep. Argentina. Email: fernandoarielmanzano@ hotmail.com

${ }^{2}$ Universidad Nacional de Córdoba. Rep. Argentina. Email: fernandoarielmanzano@ hotmail.com
}

Fecha de recepción: 4 de noviembre de 2015. Fecha de aceptación: 14 de enero de 2016. 


\section{INTRODUCCIÓN}

En este trabajo buscamos demostrar que ciertas regularidades que se presentan a escala nacional, con respecto a la participación de las personas de 14 años y más en la actividad económica, no expresan ese patrón si utilizamos información a escalas espaciales menores.

Las regularidades que tomaremos como referencia serán tres:

- La desigualdad entre varones y mujeres en las posibilidades de acceso al mundo del trabajo, expresado mediante los diferentes niveles de participación en la población económicamente activa (personas que se encuentran ocupadas o buscando empleo activamente) (NOVICK et al., 2008).

- La relación positiva entre el nivel educativo y la participación en el mercado de trabajo de las personas. Debido a que se considera que un mayor capital humano proporciona a los individuos no sólo la posibilidad de obtener salarios más elevados a lo largo de su vida activa, sino que también otorga una mayor tasa de participación y mayor estabilidad en el empleo (SALINAS JIMÉNEZ et al., 2011).

Considerando la participación en el mercado de trabajo según grupos de edad, las tasas de participación laboral más bajas se encuentran en el grupos de edades de 65 años y más, seguido del grupo de menor edad, entre 15 y 24 años, tanto para hombres como para mujeres (SALVIA et al., 2006).

\section{ALGUNAS CONSIDERACIONES METODOLÓGICAS ACERCA DE LAS CIUDADES INTERMEDIAS}

Este artículo subraya la importancia de incorporar las escalas espaciales en el análisis de la estructura ocupacional en el mercado de trabajo, ya que como explica Gutiérrez Puebla, "la adopción de distintas escalas espaciales en la investigación geográfica es la clave en ocasiones para entender la realidad en toda su complejidad" (GUTIERREZ PUEBLA, 2001).

La espacialidad social se expresa a través de su territorialidad y periodicidad. La territorialidad es la expresión de la espacialidad en contextos históricos y territoriales concretos (RANDOLPH, 1990). Además el análisis espacial permite restablecer el contexto particular del mercado laboral, y contribuye a la comprensión de los procesos socio-ambientales.

La categoría espacio tiene un valor intrínseco en el análisis de las relaciones entre actividad económica, sociedad y ambiente. Conocer las diferentes características de la estructura ocupacional a las diferentes áreas geográficas, también permite aportar información para un planeamiento de acciones de control y localización de recursos por parte de las políticas públicas.

Debemos tener en cuenta los cambios que se vienen gestando en décadas recientes, la dinámica poblacional no privilegia sólo a las grandes urbes como en el pasado, sino que también contribuye al crecimiento de las ciudades medianas o pequeñas, cuya particularidad radica en el estrecho vínculo de éstas con el mundo rural.

Las grandes ciudades hace tiempo que perdieron la capacidad de absorción de la población que migra en busca de mayores oportunidades de educación y de trabajo ${ }^{3}$ (LLONA et al., 2004). En los viejos destinos, los migrantes han dejado de percibir las garantías de un trabajo estable. Los migrantes jóvenes, deciden en gran medida, una vez finalizado su proceso de formación educativa, regresar a su lugar de origen, y mejor preparados buscar nuevas oportunidades de inserción laboral.

Por otro lado, debemos aclarar que las delimitaciones de las ciudades con rígidos criterios de cantidad resulta totalmente infructuosa. Una ciudad se considera intermedia no sólo con arreglo

\footnotetext{
${ }^{3}$ Los estudios de caso realizados en este trabajo corresponden a Chile, y demuestran que los jóvenes rurales han empezado a migrar a ciudades intermedias no sólo en busca de empleo sino también de la oferta de educación superior que allí se encuentra. Esta situación también se verifica en los datos censales -sobre todo en el último periodo intercensal-, en donde se destaca un crecimiento de las migraciones intraprovinciales en relación a las interprovinciales.
} 
a tallas demográficas y dimensiones determinadas sino, sobre todo, con relación a las funciones que desarrolla: el papel de mediación en los flujos (bienes, información, innovación, administración, etc.), su influencia sobre sus territorios urbanos y rurales, también mediante la intermediación entre los espacios locales/territoriales y los espacios regionales/nacionales e, inclusive, globales. El concepto de ciudad intermedia, que sólo puede definirse vagamente, supone realidades diversas, presentes en todos los contextos, pero considerablemente menos visibles que las que configuran las grandes aglomeraciones urbanas (BELLET SANFELIU et al., 2004).

Desde esta perspectiva, cada ciudad existe en lo que denominamos un sistema de "ramilletes urbanos", y no deben ser entendidas sin considerar las vinculaciones con las regiones rurales y con las otras ciudades con las que se complementa en sus funciones.

En estos sistemas o redes, las ciudades intermedias y pequeñas, muchas veces "invisibles" para todos, van cobrando dinamismo y fuerza, se transforman en centros de intercambio económico -fundamental para el desarrollo de algunas áreas rurales y urbanas menores-, y de interacción social y cultural, en donde se modifica significativamente la relación tradicional entre lo rural y lo urbano.

La modernización agropecuaria también ha significado un impacto en las ciudades intermedias, debido a que como mencionamos anteriormente, en la actualidad las migraciones del campo no se dirigen a las grandes ciudades y a las metrópolis necesariamente, sino que tienden a relocalizarse en pequeños poblados o ciudades medianas, sin romper el vínculo con las actividades agropecuarias -la movilidad poblacional interna entre centros urbanos menores también se ha incrementado-, (LLONA et al., 2004). Si bien el artículo de Llona hace referencia al territorio chileno, la inserción de las mejoras tecnológicas en el sector agrícola por parte de las empresas multinacionales vinculadas, son un componente que se expresa a escala global, dado que no debe restarse importancia a la nueva la división de los procesos productivos, que operan de manera integrada horizontalmente a nivel supra nacional.

Con respecto a la información estadística que utilizaremos en este artículo, corresponde al bloque ocupacional de los últimos tres Censos Nacionales de población de la Argentina, llevado adelante en los años 1991, 2001 y 2010. Conforme la matriz legalista de los censos ${ }^{4}$, es relevada sobre las personas de 14 años y más, por definir esa edad el fin del periodo escolar obligatorio para el Estado ${ }^{5}$ (OTERO, 1999). La pregunta sobre condición de actividad ${ }^{6}$ nos permite dividir en dos grupos a todas aquellas personas de 14 años y más. Un primer grupo formado por las personas que se encuentren trabajando o deseen trabajar en un momento determinado, denominados como "población económicamente activa" -en adelante PEA-y un segundo grupo formado por la población restante mayor de 14 años, que corresponde a las personas no económicamente activas -en adelante PNEA-.

Dentro de la PEA (toda la población de 14 años y más, que participa activamente en el mercado de trabajo) se suelen distinguir habitualmente dos subconjuntos: el de los ocupados -quienes desempeñan alguna actividad económica- y los desocupados -definidos como aquellos que buscan activamente un empleo-.

Con respecto al número total de desocupados, se suele considerar, en cambio, que existe una sub-representación de este conjunto, debido a que sólo distingue a aquellos que buscan trabajo,

\footnotetext{
${ }^{4}$ Por legalismo se entiende la tendencia de los sistemas estadísticos a estudiar la realidad social a partir de definiciones jurídicas codificadas por el Estado y no mediante definiciones sociológicas más sensibles a la prácticas sociales efectivas (OTERO, 1999).

${ }^{5}$ La inadecuación del precepto legalista es particularmente evidente en este punto, ya que la edad al inicio de la actividad laboral era en muchos casos inferior a la establecida por la ley. Una situación típica a este respecto, entre muchas otras referencias posibles, es ilustrada por CACOPARDO et al., (1997).

${ }^{6}$ Cabe destacar que la condición de actividad, que clasifica a la población en económicamente activa (PEA)e inactiva (PNEA), permite una aproximación al mercado de trabajo por medio de la tasa de actividad y al desempleo, a través de la tasa de desocupación. En ese sentido, la captación de la condición de actividad no es menor, ya que el método de recolección de la información se ve influida por la evaluación de los encuestadores y la percepción subjetiva de los entrevistados, y ambas a su vez se encuentran condicionadas por construcciones sociales, decisiones teóricas en la elaboración del cuestionario y estereotipos socioculturales.
} 
sin relevar a quienes se retiran del mercado laboral o no buscan trabajo, dadas las malas condiciones laborales existentes. Estos últimos constituyen la desocupación "invisible" o no manifiesta y representarían una oferta potencial de trabajadores disponibles si perciben mejoras en las condiciones del mercado de trabajo actual. Este grupo de trabajadores, metodológicamente, presenta mucha dificultad para lograr cuantificarlo (PALOMINO, 1988).

El otro componente del mercado de trabajo corresponde a la demanda de empleo, que representa el agregado de la cantidad de trabajadores que las empresas o empleadores están dispuestos a contratar. La demanda de empleo depende también de las potencialidades de la oferta de empleo, de modo tal que esta última pueda satisfacer los requerimientos necesarios en cuanto a nivel educativo, conocimiento tecnológico, antigüedad de tiempo en el oficio; así como otras características necesarias para ser incorporado al proceso productivo. Es decir, la oferta y demanda de trabajo tienen en común muchos elementos que determinan su comportamiento de manera interdependiente.

\section{PARTICIPACIÓN EN LA ACTIVIDAD ECONÓMICA SEGÚN GENERO}

De acuerdo al Censo Nacional de Población de 1991, el 56,7\% de la población de 14 años y más del país era económicamente activa (se trata de personas que se encuentran empleadas o buscando un empleo). En el año 2001, la tasa de participación en el mercado laboral fue de $57,2 \%$, y en el año 2010 alcanzó la cifra record de $65,0 \%$.

La TABLA $\mathrm{N}^{\circ} 1$ refiere a datos a nivel nacional. Se destacan los condicionantes relativos al género en el nivel de participación en el mercado laboral. En el año 1991 los diferenciales según sexo, presentan una tasa de participación de los hombres que resultaba prácticamente el doble que la tasa femenina (CONTARTESE et al., 2005, p. 138).

Esta diferencia presenta una fuerte tendencia descendente en los últimos 20 años. En el 2001 la brecha en la participación económica entre hombres y mujeres era de 25,6 puntos porcentuales (en adelante p.p.), y el último dato censal del año 2010 presenta una brecha de 22,8 p.p., -la variación relativa intercensal 2001-2010, en la tasa de participación masculina fue de tan sólo 2,2\%, mientras que en las mujeres el incremento alcanzó una cifra de 36,3\%-.

\section{TABLA 1}

Tasa de participación en la actividad económica, según sexo. Población de 14 años y más. Total del país. Años 1991, 2001 y 2010

\begin{tabular}{|c|c|c|c|}
\hline Año & Total & Varon & Mujer \\
\hline $\mathbf{1 9 9 1}$ & 56,7 & 75,1 & 39,6 \\
\hline $\mathbf{2 0 0 1}$ & 57,2 & 70,5 & 44,9 \\
\hline $\mathbf{2 0 1 0}$ & 65,0 & 76,8 & 54,0 \\
\hline
\end{tabular}

Fuente: Elaboración propia en base a los Censos Nacional de Población y Vivienda 1991, 2001 y 2010 (INDEC).

Teniendo en cuenta los diferentes niveles de escala espacial -regiones, provincias y localidades-, se perciben variaciones crecientes con respecto al valor de la media nacional conforme aumentamos el nivel de desagregación de la información utilizada.

En el 2010, a nivel regional, la brecha entre género en la participación laboral más alta fue de 27,6 p.p. en la región Noroeste y la menor ha sido de 20,6 p.p. en la región Metropolitana. Es decir, estos valores se encuentran a una distancia de 2,2 p.p. por debajo del promedio nacional y 4,8 p.p. por encima.

Si incorporamos los valores de la tasa de participación laboral según sexo a nivel provincial, se presenta una brecha máxima entre 41,6 p.p. en Santiago del Estero y una diferencia mínima de 15,8 p.p. en la Ciudad Autónoma de Buenos Aires. Los valores provinciales se encuentran 7,0 p.p. por debajo y 18,8 p.p. por encima del valor de la brecha nacional (ver Figura $\mathrm{N}^{\circ} 1$ ). 
FIGURA 1

Diferencia en la tasa de participación masculina menos femenina (en p.p.). Población de 14 años y más. Total del país. Año 2010.

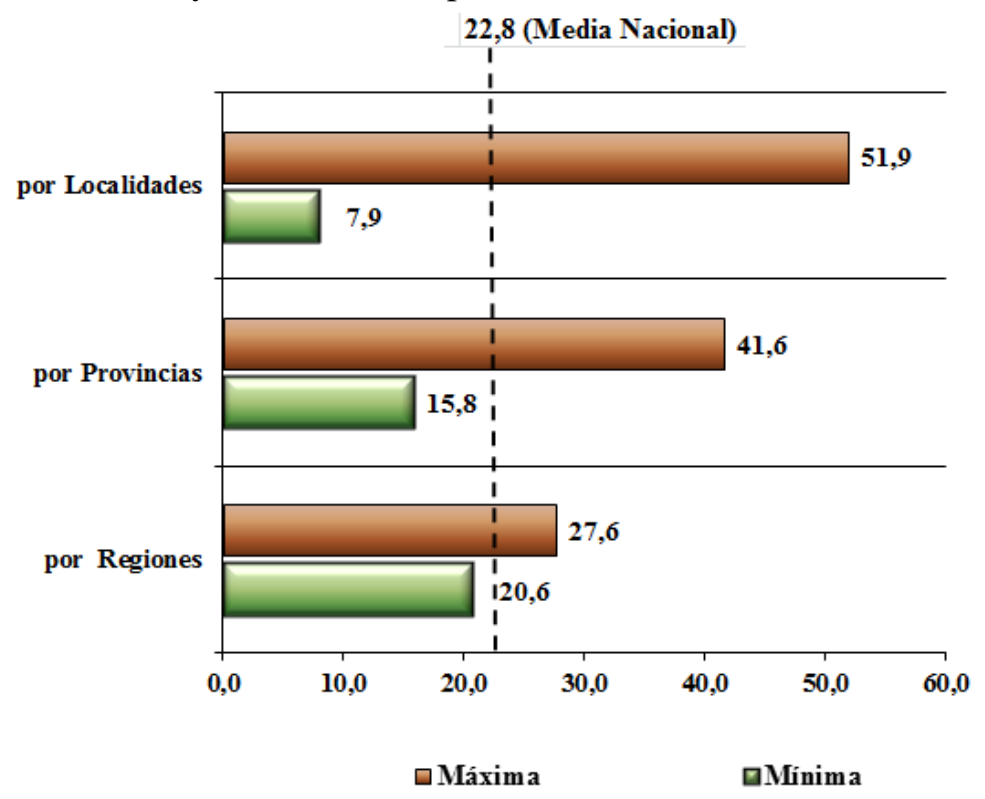

Fuente: Elaboración propia en base al Censo Nacional de Población y Vivienda 2010 (INDEC).

A continuación disminuiremos el nivel de escala espacial de nuestro análisis. Consideraremos a los aglomerados y las localidades que poseen más de 2.000 habitantes, estas alcanzan un total de 958 aglomerados urbanos. Los mismos pueden clasificarse según las siguientes categorías urbanas: 3 Metrópolis o Ciudades Grandes -con población superior 1 al millón habitantes-; 9 Aglomerados grandes -su población total supera 400 mil y es inferior al millón de habitantes-, 53 Aglomerados Medios -con población entre 50 mil habitantes y 400 mil-, 111 Aglomerados Pequeños -que poseen más de 20 mil habitantes y menos de 50 mil-y 782 Pueblos -con población entre 2.000 y 20 mil habitantes-.

Las jerarquías urbanas presentan una relación inversa con la variabilidad de la brechas de participación laboral según género. La dispersión en las Aglomerados Grandes utilizando como medida el coeficiente de variación (en adelante C.V.) es de 11,9\%, las Aglomerados Medios tienen un C.V. de 13,7\%, las Aglomerados Pequeñas poseen un C.V. de 14,9\% y en los Pueblos grandes se alcanza un valor de C.V. de 20,6\%-. En esta última categoría urbana encontramos las localidades con valores más alejados con respecto a la brecha a escala nacional. En la localidad de "Villa Los Coihues", perteneciente a la provincia de Rio Negro, encontramos una brecha en la participación según sexo de tan sólo 7,9 p.p. y en la localidad "El Aguilar", ubicada en la provincia de Jujuy se encuentra la brecha máxima, con un valor de 51,9 p.p.

\section{PARTICIPACIÓN SEGÚN NIVEL EDUCATIVO}

Suele considerarse una clara asociación positiva entre el nivel educativo y la participación en el mercado de trabajo: a medida que aumenta el primero se incrementa la segunda (VILLALOBOS MONROY et al., 2009). Este comportamiento se observa tanto entre varones como en mujeres.

A nivel nacional, la brecha en el nivel de participación masculina y femenina antes mencionada, se va reduciendo a medida que el nivel de instrucción aumenta. Por otra parte, entre las mujeres la educación juega un papel más destacado que entre los varones para condicionar su participación en el mercado laboral: entre ellas, la tasa de actividad económica de las más instruidas casi duplica a la de sus congéneres con menor nivel de educación, 81,8\% versus $44,5 \%$; mientras que entre los varones son del $91,3 \%$ y $77,0 \%$, respectivamente. 
FiguRA 2

Tasa de participación en la actividad económica por sexo y nivel de educación. Población de 30 años y más. Total del país 2010.

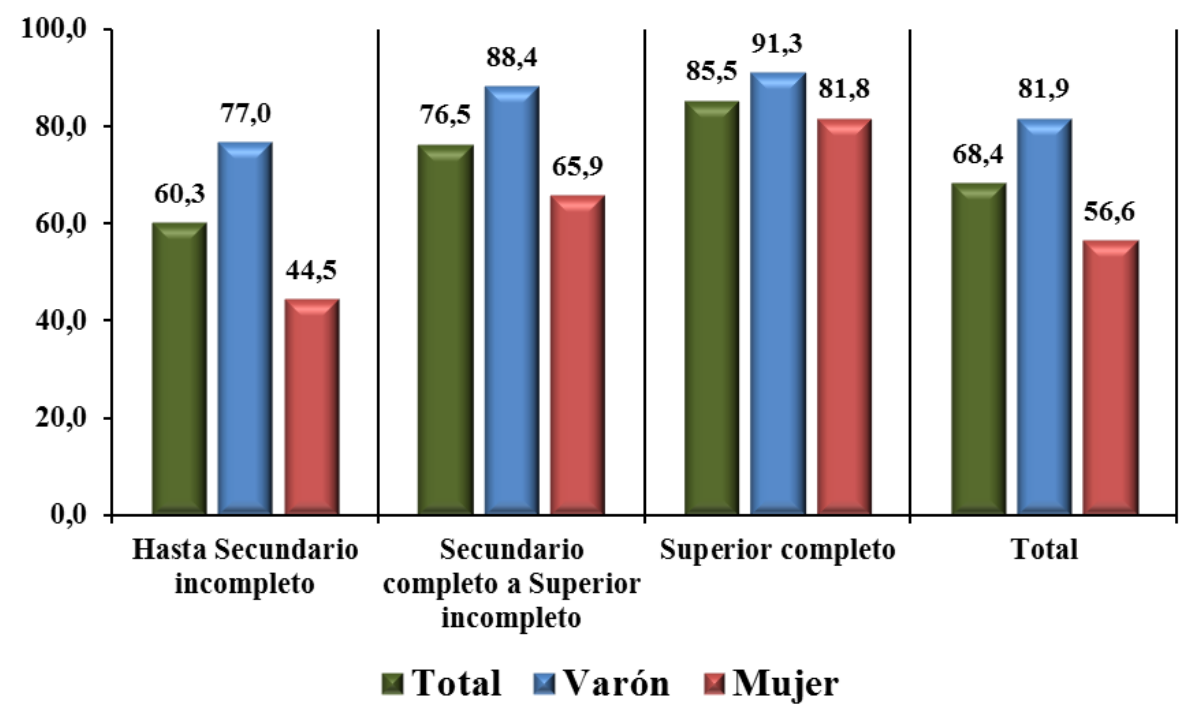

Fuente: Elaboración propia en base al Censo Nacional de Población y Vivienda 2010 (INDEC).

Con respecto a la Tasa de Actividad de la población de 30 años y más, según nivel educativo, observamos que el promedio nacional en el año 2010 presenta menor participación de la mujer con nivel de estudios hasta secundario completo, con 32,5 p.p. menos que los hombres, y en el caso del nivel de estudios superiores completos esta brecha se reduce a 9,5 p.p.

Teniendo en cuenta las diferencias regionales en el año 2010, las brechas de género en la participación de la fuerza de trabajo con nivel de estudios hasta secundario incompleto presenta un mínimo de 21,8 p.p. en la región de Cuyo y un máximo de 37,4 en el Noreste. Es decir, 10,7 p.p. menos -o una disminución relativa de $32,8 \%$ - que la media nacional y 4,9 puntos por encima -o un aumento relativo de $15,2 \%-$, respectivamente. Tal como se observa en la Figura $\mathrm{N}^{\circ} 3$.

En el caso de la fuerza de trabajo con niveles de estudios superiores completos, la brecha de género presenta un mínimo de tan sólo 4,7 p.p. nuevamente en la región de Cuyo y se alcanza la diferencia más grande en la región Pampeana, de 11,0 p.p. Las diferencias mencionadas con respecto a la media nacional, son de 4,8 p.p. por debajo -o una disminución relativa de $50,3 \%$ y 1,5 p.p. por encima -o un aumento relativo de $15,8 \%-$.

$\mathrm{Si}$ analizamos a nivel provincial, la brecha de género en la fuerza de trabajo con nivel de estudios menor a secundario completo, es máxima en la provincia de Mendoza con un valor de 40,0 p.p. y mínima en San Juan, presentando una diferencia negativa de 20 p.p. - es decir, la tasa de participación femenina supera a la masculina, con valores de 57,1\% y 37,1\%, respectivamente-.

Con respecto a la participación de los mayores de 30 años con nivel de estudios superiores completos, la brecha de género máxima es de 12,6 p.p. en la provincia de Tucumán y la menor diferencia se da nuevamente en San Juan de $-4,3$ p.p. Por tanto, se detecta que a escala provincial existen valores de participación femenina en la actividad económica superiores a la masculina, independientemente del nivel de estudios logrados.

A nivel de localidades, la brecha de género en la participación laboral de la población mayor de 30 años con estudios secundarios incompletos o menos, presenta su valor máximo de 71,3 p.p. en la localidad de "El Aguilar" -provincia de Jujuy-y la mínima es de -24,1 p.p. en "Villa General San Martín", perteneciente a la provincia de San Juan. 
FIGURA 3

Brechas en Tasa de participación masculina menos femenina (en p.p.). Población de 30 años y más. Total del país 2010.

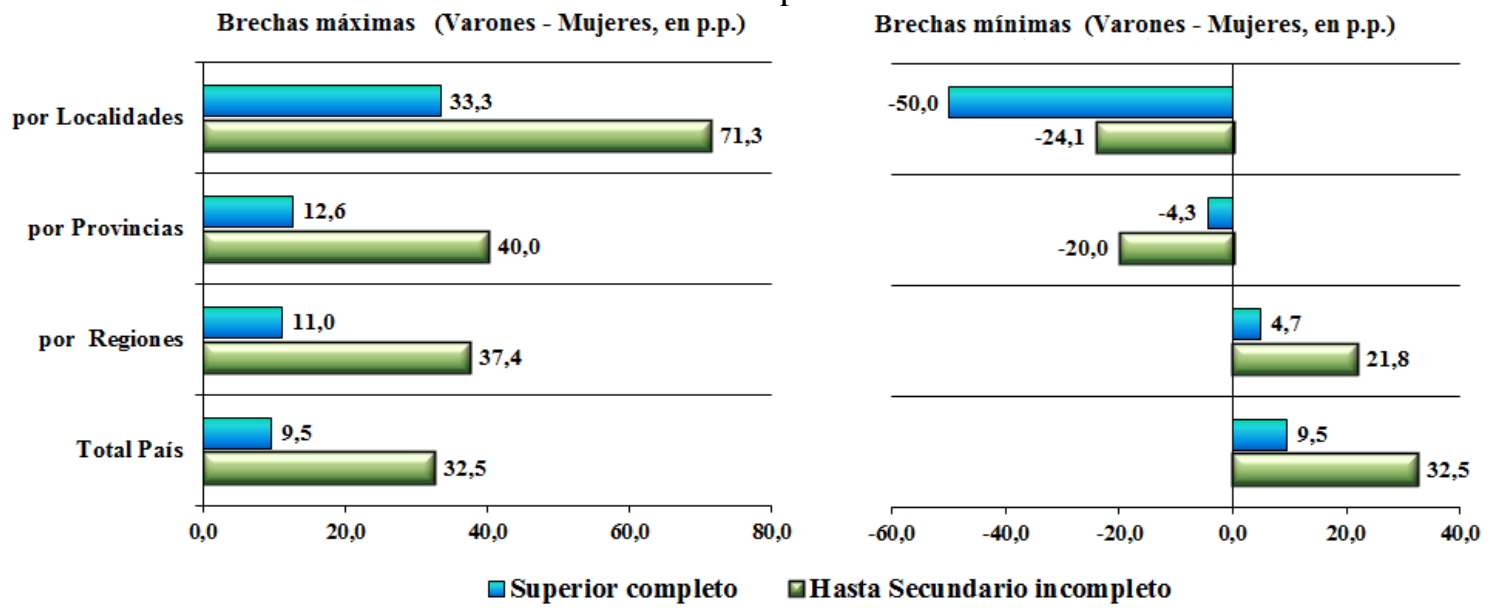

Fuente: Elaboración propia en base al Censo Nacional de Población y Vivienda 2010 (INDEC).

Con respecto a la brecha de género en las fuerzas laborales con estudios superiores completos a nivel localidad, la diferencia máxima es de 33,3 p.p. en la localidad de "Las Perlas" en la provincia de Rio Negro, y la menor brecha es de -50,0 p.p. en la localidad "La Verde" en la provincia de Chaco -es decir, la tasa femenina alcanza el 100\% y la masculina es de 50\%-.

A continuación analizaremos la composición al interior de cada sexo, así como la distribución según niveles educativos máximos alcanzados en ambas fuerzas laborales considerando sólo la población mayor a 30 años-.

En la Figura $N^{\circ} 4$ se observa que a escala nacional para el año 2010, las mujeres tienen menor proporción de población activa mayor a 30 años con nivel educativo hasta secundario incompleto en relación con los hombres, así como también mayor peso relativo de población activa -mujeres que se encuentran empleadas o buscando un empleo de manera activa-, con estudios superiores completos. La fuerza de trabajo femenina presenta 12,8 p.p. menos de trabajadoras con nivel educativo hasta secundario incompleto, y 11,0 p.p. más de trabajadores con niveles de estudios superiores completos, en relación con los hombres en actividad económica.

Teniendo en cuenta la división regional en el año 2010, las diferencias entre género en la proporción de fuerza laboral con nivel secundario incompleto o menos, varía de un mínimo de 8,5 p.p. en la región de Cuyo y un máximo de -17,1 p.p. en el Noreste (el cálculo surge de restar la tasa de participación femenina menos la masculina). En las mismas regiones también se presentan diferencias extremas en el porcentaje de población activa con estudios superiores completos entre sexos. La brecha presentan un valor mínimo de sólo 7,1 p.p. en la región de Cuyo y una diferencia máxima de 14,0 p.p. en la región Noreste a favor de las mujeres. Tal como se observa en la Figura $\mathrm{N}^{\circ} 5$.

Incrementando el análisis espacial a escala provincial se observan situaciones en donde la fuerza de trabajo femenina no se encuentra más capacitada que la masculina.

En la provincia de San Juan, la población activa femenina presenta 9,5 p.p. más de estudios secundarios incompletos en relación a la masculina, y 8,0 p.p. menos de trabajadores femeninos con estudios superiores completos que la PEA masculina.

Considerando a nivel de localidades, encontramos un valor máximo de 12,8 p.p. más de población activa femenina (trabajadoras ocupadas o en busca de un empleo) en relación a la masculina, con el menor nivel de instrucción -hasta secundario incompleto-, en la localidad de "Caucete" -provincia de San Juan-. En esta misma localidad también se encuentra la diferencia más grande de participación de trabajadores con estudios superiores completos entre las fuerzas laborales de ambos sexos, de 14,5 p.p. a favor de los varones. 
FIGURA 4

Composición porcentual de la Fuerza de trabajo por sexo y nivel de educación. Población de 30 años y más. Total del país 2010.

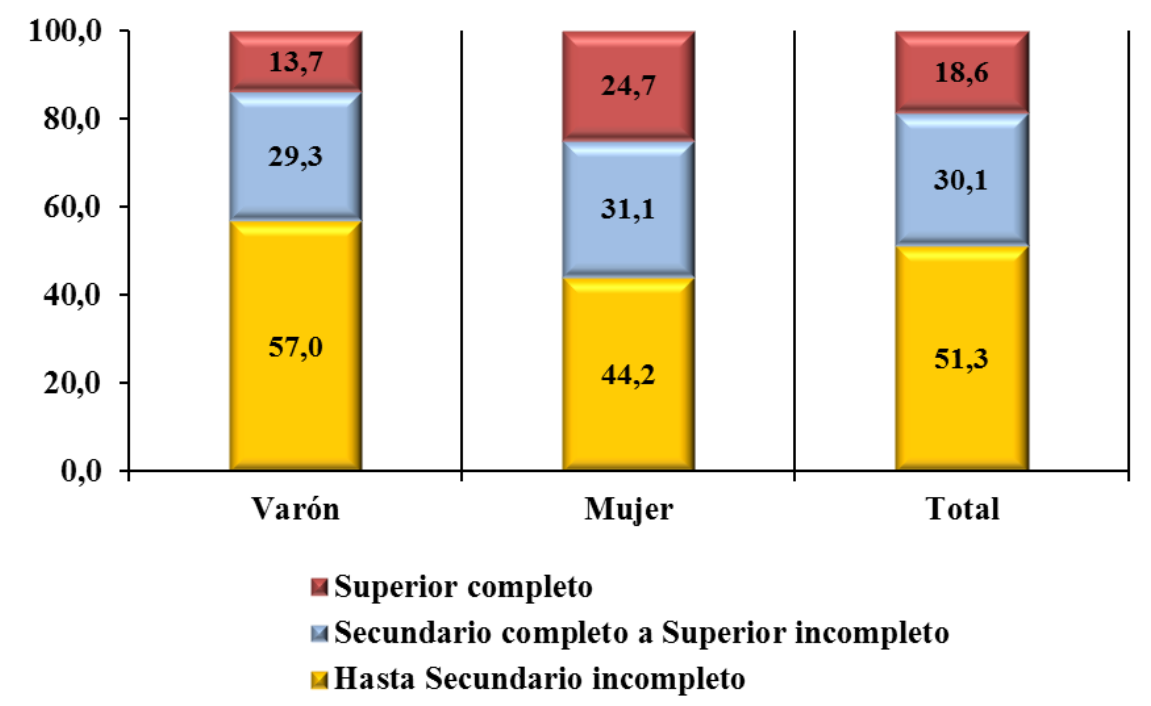

Fuente: Elaboración propia en base al Censo Nacional de Población y Vivienda 2010 (INDEC).

FIGURA 5

Diferencia porcentual máxima (Mujeres - Varones) en la fuerza de trabajo según nivel educativo (en p.p.). Población de 30 años y más. Total del país 2010.

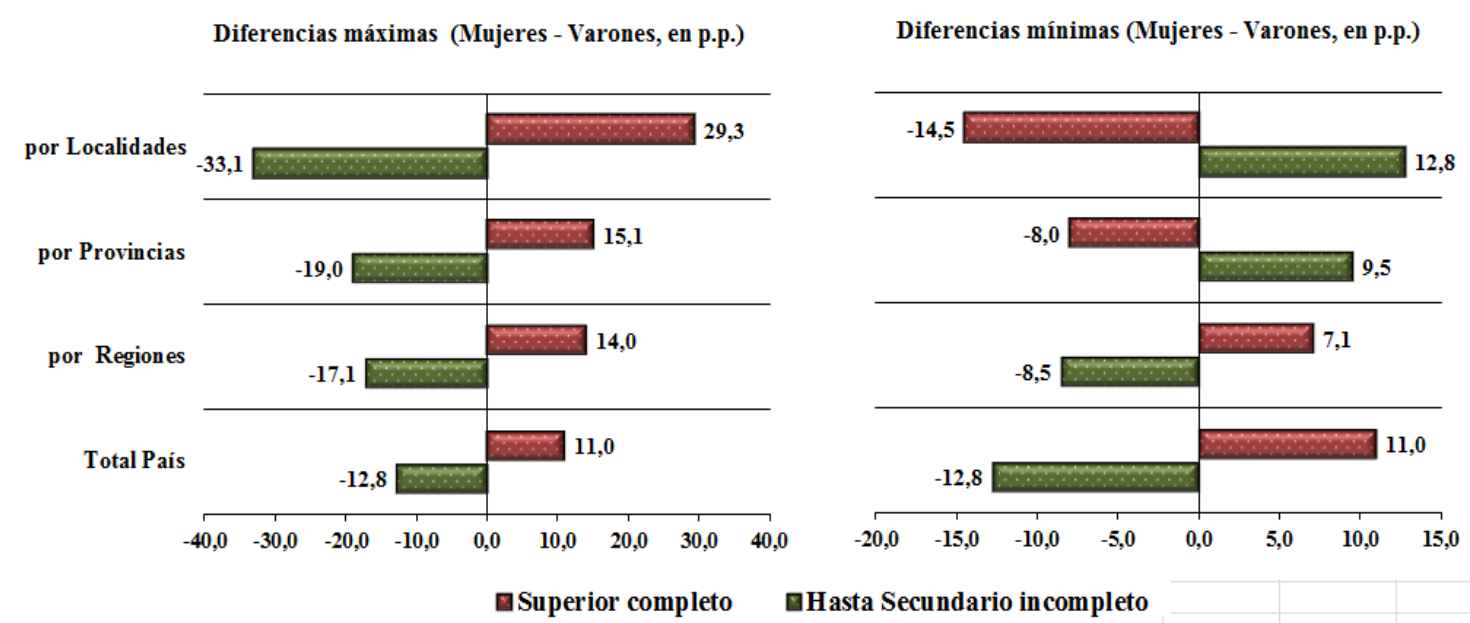

Fuente: Elaboración propia en base al Censo Nacional de Población y Vivienda 2010 (INDEC).

Antes de finalizar este apartado nos queda atender nuestro segundo objetivo, que hace referencia a la relación positiva entre el nivel educativo y la participación en el mercado de trabajo para ambos sexos.

Considerando información agregada a escala provincial, se verifica la relación positiva entre nivel educativo y participación en la actividad económica (personas que se encuentran trabajando o buscando un empleo activamente).

Pero si incrementamos la escala de análisis espacial empiezan a surgir casos que contradicen esta premisa, como los que anunciaremos a continuación.

Dentro de los Aglomerados Medios, encontramos que en las localidades de Clorinda, San Ramón de la Nueva Orán, Luján, y en los aglomerados de Villa Carlos Paz -San Antonio de Arredondo y Viedma - y Carmen de Patagones, la tasa de participación económica de los varones con nivel de instrucción secundario completo supera a la de los que poseen estudios superior completo. 
En los Aglomerados Pequeños ocurre lo mismo en la localidad de Pinamar, y en los aglomerados La Falda -Huerta Grande-, Valle Hermoso y Cosquín -Santa María de Punilla- y Bialet Massé.

Considerando los pueblos de más de 2.000 habitantes, en las localidades de La Verde, San Pedro de Colalao y Gaboto, los valores de la tasa de participación masculina de trabajadores con secundario incompleto superan a la tasa de participación de los que poseen secundario completo.

De los 782 pueblos con más de 2.000 y menos de 20.000 habitantes, en 129 pueblos $-16,5 \%$ de los casos-, la tasa de participación de los trabajadores con nivel secundario completo supera a la tasa de actividad de los que poseen nivel superior completo.

En contraposición, entre la PEA femenina, sólo encontramos un caso en donde la tasa de participación de las trabajadoras con nivel secundario completo supera a las que poseen estudios superiores completos, ubicado en la localidad de "Juan Bautista Alberdi".

\section{RELACIÓN ENTRE NIVELES DE ACTIVIDAD Y LA EDAD}

Al examinar los niveles de actividad por sexo y edad que aparecen en la Figura $\mathrm{N}^{\circ} 6$, se observa que los hombres participan con mayor intensidad en el mercado laboral, con independencia de la edad. Las diferencias de género se agudizan justamente en las edades centrales, entre los 20 y los 65 años, para atenuarse al inicio y al final de la vida activa.

Aún las mujeres de 25 a 49 años, que alcanzan nivel máximo de participación con tasas que superan el $50 \%$, mantienen desventajas notorias con respecto a los varones de esas edades, entre los cuales más del $90 \%$ está inserto en el mercado de trabajo.

Hay dos hechos que vale la pena señalar: por un lado, el crecimiento entre 1991 y 2010 de la participación femenina para todos los grupos de edad y la disminución de la participación masculina entre los 25 y 49 años. Por otro, la alta proporción de población que permanece activa en el grupo etario de 65 a 74 años; especialmente el incremento de las mujeres en los últimos 20 años, el incremento en la tasa de actividad fue de $65,2 \%$, mientras que en el de los hombres fue de 41,9\% -si bien el crecimiento de la participación del grupo de 75 años y más entre 1991 y 2010 fue el de máximo crecimiento en las mujeres, también lo fue en los hombres, por tanto, la tasa de actividad de las primeras continua siendo la mitad que la masculina-.

FIGURA 6

Tasa de participación en la actividad económica por sexo y grupo de edad. Total País. Años: 1991,2001 y 2010.

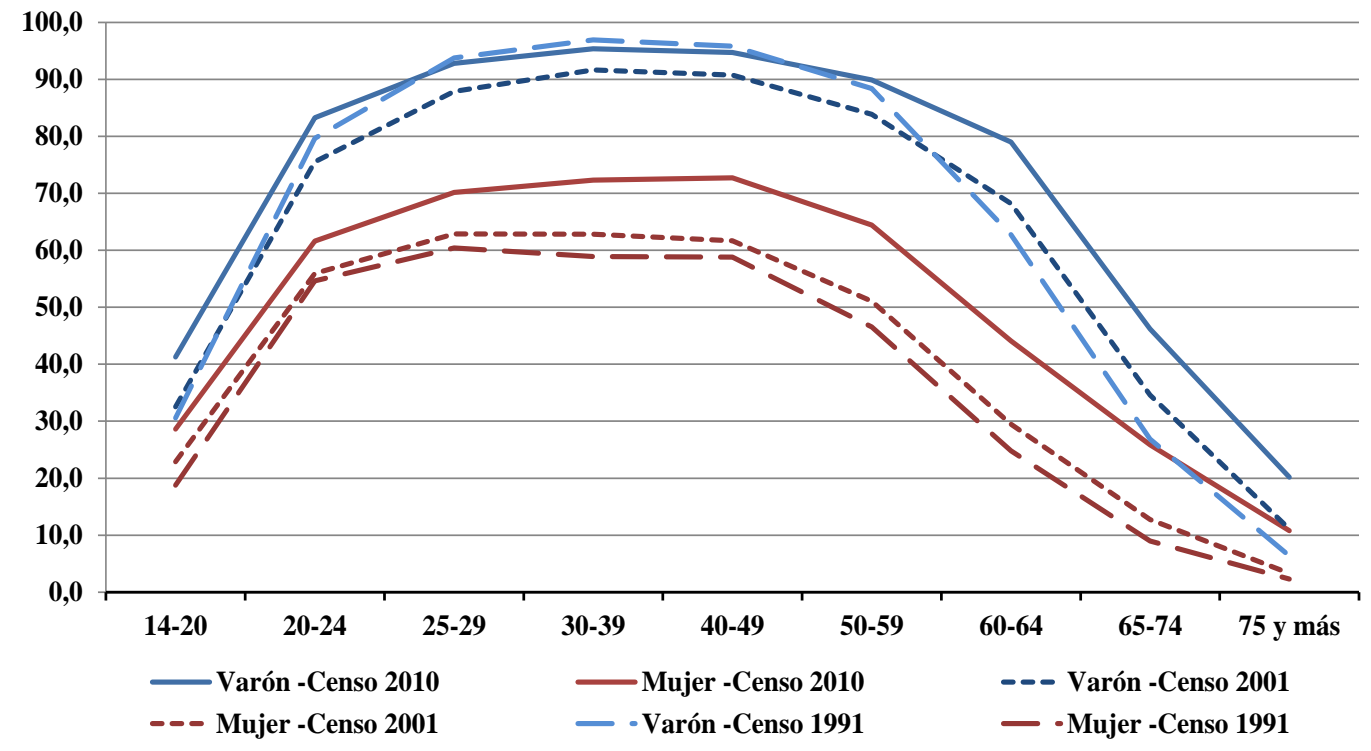

Fuente: Elaboración propia en base a los Censos Nacional de Población y Vivienda 1991, 2001 y 2010 (INDEC). 
Tal como se observa en la Figura $\mathrm{N}^{\circ}$, la tasa de participación entre los grupos de edad de 14 a 24 años y el siguiente de 25 a 29 años, presentó un incremento de 34,0 p.p. en los hombres y de 27,3 p.p. en las mujeres. Entre este último grupo de edad y el de 30 a 49 años, la tasa de actividad aumentó sólo 2,7 p.p. y 2,3 p.p., para varones y mujeres, respectivamente.

A escala nacional, en ambos sexos se presenta la mayor tasa de participación en el grupo de 30 a 49 años, luego esta última comienza a descender. Si comparamos la tasa de actividad del grupo de 30 a 49 años con respecto al de 50 a 64 años, este último disminuye en 8,6 p.p. en el caso de los hombres y 14,4 p.p. en las mujeres. Entre el grupo de 50 a 64 años y el de 65 y más, se presenta la mayor disminución (50,6 p.p. y 38,6 p.p., en varones y mujeres, respectivamente).

En síntesis a nivel del total del país se verifica un crecimiento en la tasa de participación en la actividad económica (personas ocupadas o buscando un empleo de manera activa) entre los 14 hasta 49 años, y luego comienza a descender el nivel de participación.

FIGURA 7

Estructura de la tasa de participación económica por grupo de edad y sexo. Total del país. Año 2010.

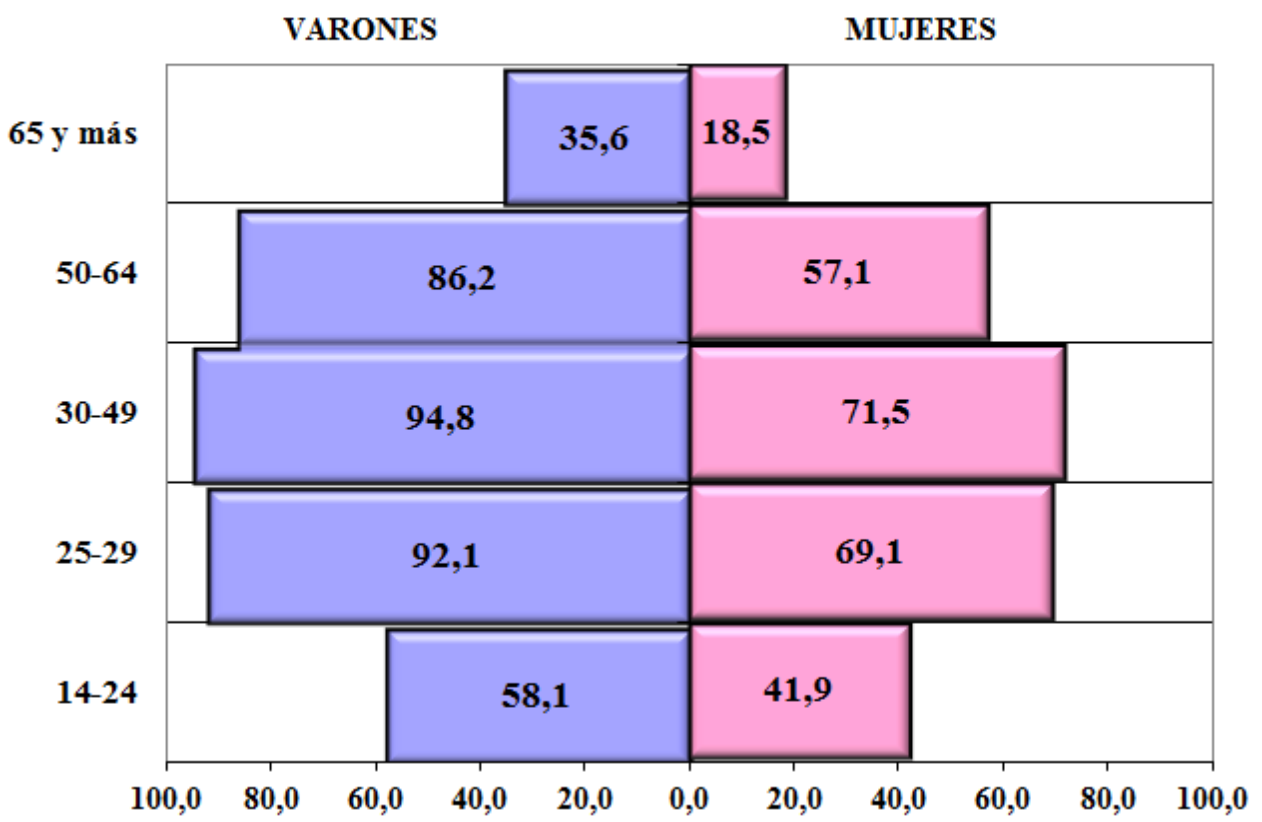

Fuente: Elaboración propia en base a los Censos Nacional de Población y Vivienda 2010 (INDEC).

A nivel regional, en el Área Metropolitana encontramos que las mujeres del grupo de edad de 25 a 29 años, presentan mayor tasa de participación laboral que el grupo etario de 30 a 49 años.

A nivel provincial nos encontramos que esta situación de mayor participación en la actividad económica del grupo de edad de 25 a 29 en relación con el grupo de 30 a 49 años, se observa en los varones en la provincia de Misiones y en las mujeres en la Ciudad Autónoma de Buenos Aires.

En los aglomerados y localidades correspondientes a diferentes categorías urbanas, encontramos una cantidad más importante de casos que contradicen esta premisa, que suele ser validada en todos los manuales, mediante la representación gráfica de una "U" invertida para explicar la participación laboral en función de la edad.

Dentro de los Aglomerados Medios, encontramos en los hombres que el grupo activo de 25 a 29 años, presenta mayor tasa de participación laboral que el grupo etario de 30 a 49 años en las localidades de Pergamino, Junín, San Francisco Frontera, Venado Tuerto y Ushuaia. Y esta 
situación en las mujeres ocurre en las localidades de: Goya, Bahía Blanca, Tandil, Punta Alta, Azul, San Francisco Frontera, Rafaela, Puerto Madryn, y en los aglomerados de Gran Río Cuarto, Villa Carlos Paz-San Antonio de Arredondo.

En los Aglomerados Pequeños, la situación de mayor participación en la actividad económica del grupo de edad de 25 a 29 años en relación al grupo de 30 a 49 años, se verifica en los hombres en tres localidades (Chajarí, San Vicente y Puerto Deseado), en las mujeres en 9 centros (Lobos, Coronel Suárez, Saladillo, Las Flores, Río Tercero, Villa General San Martín, Casilda, Cañada de Gómez y Río Grande), y en ambos sexos en 2 localidades (Arroyito y Arroyo Seco).

De los 782 pueblos entre 2.000 y 20.000 habitantes encontramos casos donde la tasa de participación del grupo de 50 a 64 años, supera al grupo de 30 a 50 años. Esto es así, en el caso de los hombres, en dos localidades (Villa Basilio Nievas y General Ballivián) y en el de las mujeres en cinco núcleos (Gancedo, La Verde, El Aguilar, Colonia El Simbolar y Garmendia).

Dentro de esta categoría urbana, encontramos 152 pueblos (19,4\% del total) para el caso de los hombres y 150 localidades $(19,2 \%)$ para el de las mujeres en donde el grupo activo de 25 a 29 años, presenta mayor tasa de participación laboral que el grupo etario de 30 a 49 años.

\section{CONCLUSIONES}

En este trabajo mostramos que ciertas pautas generales sobre la participación en la actividad económica (personas que se encuentran empleadas o buscando empleo de manera activa) según género, nivel de instrucción y edad obedecen más a la agregación estadística de diferentes realidades urbanas y regionales que a pautas "indiscutibles" para cualquier contexto económico y social.

Si bien la participación femenina en la PEA (mujeres ocupadas o en busca de un empleo) se ha incrementado, todavía no existe una convergencia (parte de este hecho puede deberse al subregistro del trabajo femenino a pesar de los esfuerzos en este sentido). Sin embargo, en algunos contextos de la argentina esa participación resulta mayor.

También se espera que el incremento del nivel de instrucción aumente las posibilidades de inserción en la PEA (personas ocupadas o bien ofreciendo en el mercado su fuerza de trabajo). Esto, una vez más, no resulta válido en algunos contextos en donde su peculiar estructura económica y social no requiere de la incorporación de trabajadores calificados.

Por último, las edades de ingreso y egreso de la PEA hacen que en general la tasa máxima se alcance entre los 30 y 65 años. Sin embargo en determinados contextos regionales esto no es así ya que se detecta mayor incorporación precoz (por debajo de los $30 \mathrm{o}$, incluso de los 20) y permanencia en el mercado de trabajo a edades más avanzadas (¿menor cobertura previsional? ¿Escasos ingresos? ¿Aburrimiento?).

\section{REFERENCIAS BIBLIOGRÁFICAS}

BELLET SANFELIU, C. y LLOP-TORNÉ, J.M. (2004): Ciudad y territorio. "Ciudades intermedias. Entre territorios concretos y espacios globales", vol. 36, n. 141-142, p. 569-58.

CACOPARDO, C. y MORENO, J. L. (1997): Poblaciones argentinas. Estudios de demografía diferencial. "Cuando los hombres estaban ausentes: la familia del Interior de la Argentina decimonónica”. En Otero, H. y Velázquez, G. (comps.), PROPIEP (CIG-IEHS), Universidad Nacional del Centro, Tandil.

CONTARTESE, D. Y MACEIRA, V. (2005): Relaciones laborales, territorios y grupos particulares de actividad. "Diagnóstico sobre la situación laboral de las mujeres". Serie, Trabajo, Ocupación y Empleo $\mathrm{n}^{\circ}$ 3. Disponible en: http://www.trabajo.gob.ar/left/estadisticas/descargas/toe/toe $03 \quad 05$ situacionLaboralMujere s.pdf [consulta: 21 de septiembre de 2015]

GUTIÉRREZ PUEBLA, J. (2001): Estudios GeoGráficos. "Escalas espaciales, escalas temporales", vol. $62, \quad \mathrm{n}^{\circ} \quad 242, \quad$ p. 89-104. Disponible en: http://estudiosgeograficos.revistas.csic.es/index.php/estudiosgeograficos/article/view/295/29 $\underline{4}$ [consulta: 17 de agosto de 2015] 
LLONA, M; RAMÍREZ CORZO, D. y ZOLEZZI, M. (2004): Las ciudades en el Perú. "Las ciudades intermedias: su rol en el desarrollo del país". DESCO, Serie: Perú Hoy, nº.

NOVICK, M; ROJO, S. y CASTILLO, V. (comp.) (2008): El trabajo femenino en la post convertibilidad. Argentina 2003-2007. Cepal. Disponible en: http://www.trabajo.gob.ar/downloads/biblioteca libros/el trabajo femenino.pdf [consulta: 3 de septiembre de 2015]

OTERO, H. (1999): Población y relaciones sociales en la campaña de Buenos Aires. "Legalidad jurídica y legalidad estadística en el paradigma censal argentino, 1869-1914". En Fradkin, R.; Cañedo, M. y Mateo, J. (comp.). GIHRR-UNMdP, Mar del Plata

PALOMINO, H. (1988): Cambios ocupacionales y sociales en Argentina.1947-1985. CISEA, Buenos Aires.

RANDOLPH, R. (1990). Configuraçao e organizaçao territorial: análise de espacialidade e temporalidade. Cad IPPUR

SALINAS JIMÉNEZ, J; SALINAS JIMÉNEZ, M. y RAHONA LÓPEZ, M. (2011): Gasto en educación, rendimientos educativos y mercado de trabajo: algunas consideraciones sobre el caso español. En XXIII Seminario regional de Política Fiscal -CEPAL-. Disponible en: http://www.cepal.org/ilpes/noticias/paginas/1/41751/salinas_rahona_salinas_doc.pdf [consulta: 3 de septiembre de 2015]

SALVIA, A y TUÑ́N, I. (2006): Encrucijadas. "Los jóvenes y el mundo del trabajo en la Argentina actual: Joven argentino", $\mathrm{n}^{\circ} 36$. Disponible en: Repositorio Digital Institucional de la Universidad de Buenos Aires: http://www.uba.ar/encrucijadas/marzo_6/notas.htm\#5 [consulta: 13 de diciembre de 2015]

VILLALOBOS MONROY, G. y PEDROZA FLORES, R. (2009): Tiempo de Educar. "Perspectiva de la teoría del capital humano acerca de la relación entre educación y desarrollo económico", vol. 10, $\mathrm{n}^{\circ}$. 20, p. 273-306. Disponible en: http://www.redalyc.org/pdf/311/31112987002.pdf [consulta: 3 de septiembre de 2015] 